\title{
Dimensión afectiva y estrategias de motivación en la enseñanza de Español Lengua Extranjera en un contexto universitario
}

\author{
Beatriz Calvo Martín
}

\begin{abstract}
Resumen:
En el contexto actual, en el que el inglés es ya la lingua franca que vehicula la motivación intrínseca y extrínseca de nuestros estudiantes, ¿en qué lugar se encuentra la motivación por el aprendizaje del español como lengua extranjera?

La motivación es un factor fundamental para el aprendizaje en general, y para la adquisición de las lenguas extranjeras en particular. Uno de los mayores potenciadores de la motivación es el desarrollo de un clima afectivo positivo, que fomente la autonomía, la cooperación y la implicación personal para lograr la efectiva adquisición de la lengua por parte de los aprendientes. Como han demostrado numerosos estudios, y en particular los de Jane Arnold, la parte afectiva del aprendizaje, muy ligada a las emociones, no se opone a la parte cognitiva, sino que, por el contrario, si ambas trabajan juntas el proceso de aprendizaje se construye sobre una base más sólida y resulta más eficaz. Se trata de considerar al aprendiente de forma holística, aunando lo cognitivo, lo emocional y lo físico. Sin motivación no hay aprendizaje, y esta depende tanto de factores individuales como relacionales, es decir, que es fundamental tomar en consideración lo que sucede dentro de y entre las personas que se encuentran en el aula. Por su parte, el Marco Común Europeo de Referencia (MCER, 7.3.1.2) reconoce la importancia de la motivación, junto con la implicación y la empatía, entre los factores afectivos esenciales para poder llevar a cabo tareas de aprendizaje de forma exitosa. Pero, ¿cómo diseñar tareas que estimulen los factores afectivos positivos haciendo que sean a la vez motivadoras, eficaces y significativas?
\end{abstract}

En esta comunicación reflexionaremos sobre la influencia que la dimensión afectiva tiene en el proceso de aprendizaje de las lenguas, y su papel para fomentar la motivación. De manera específica, nos detendremos en el caso del español como lengua extranjera. Asimismo, propondremos algunas estrategias de motivación que se pueden desarrollar a través de actividades y tareas concretas que parten de la experiencia real en un contexto universitario.

Palabras clave: motivación, dimensión afectiva, enseñanza de lenguas, español como lengua extranjera

\begin{abstract}
:
In the present context, in which English is the lingua franca that conveys the extrinsic and intrinsic motivation of our students, where is the motivation for learning Spanish as a foreign language?

Motivation is a major factor for learning in general, and more specifically for the acquisition of foreign languages. One of the greatest motivation enhancers is the development of a positive affective climate, which encourages autonomy, cooperation and personal implication in order to achieve effective acquisition. As numerous studies have shown, and particularly those of Jane
\end{abstract}


Arnold, the affective part of learning, closely linked to emotions, is not opposed to cognition, but, on the contrary, if both work together the learning process is built on a more solid basis and becomes more effective. The secret lies in developing an integrative holistic approach, focusing on cognitive, emotional and physical aspects of our learners. Learning is not possible without motivation, and it depends both on individual and relational factors, so we need to consider what happens inside but also among people in our classroom.

The Common European Framework of Reference (CEFR, 7.3.1.2) refers to motivation as one of the affective factors, together with involvement and empathy, essential to successful learning task completion. But, how to design motivating, meaningful and effective tasks by stimulating positive affective factors?

In this paper, we will reflect on the influence of affective factors on the language learning process, and their specific role in fostering motivation. We will focus on Spanish as a foreign language, analysing some motivational strategies, tasks and activities drawn from real experience in an academic context in Higher Education.

Key words: motivation, emotional dimension, language learning, Spanish as a foreign language

\section{Introducción}

En el contexto de nuestra sociedad actual, la enseñanza en general y la de las lenguas en particular ha sufrido una profunda transformación. Los profesores de lengua no podemos obviar la importancia de las herramientas digitales, que nos llevan cada vez más, y con un salto cualitativo desde la experiencia debida al COVID-19, hacia un aprendizaje mediado por la tecnología, y cada vez más realizado a distancia y de manera autónoma por los aprendientes. Sin embargo, cabe preguntarse cuál es y será el papel del profesor en este nuevo escenario de enseñanza-aprendizaje. ¿Debemos limitar nuestro papel al de ser meros gestores del aprendizaje?

Lejos de una mercantilización de la relación enseñante-aprendiente convertidos en gestor-cliente, proponemos con Trujillo Sáez (2015) un cambio de paradigma: desde el modelo que aboca a los profesores a ser meros proveedores de servicios, a un modelo en el que el objetivo sea proveer experiencias memorables de aprendizaje. Se trata de volver a considerar la enseñanza como la experiencia humana que siempre fue. Y es que nunca está de más subrayar que la enseñanza es una experiencia profundamente humana, no es posible hacer abstracción de esta dimensión humanística. De este modo, la experiencia global de aprender un idioma consiste no solo en aprender, sino también en ser, hacer y disfrutar. ¿Es posible mantener esta perspectiva humanística y ponerla en práctica en la enseñanza universitaria?

En nuestra opinión, no solo es posible sino también deseable. 
¿Cómo se consigue crear una experiencia memorable de aprendizaje? La clave está en la motivación y en tener en cuenta la dimensión afectiva del aprendizaje.

\section{¿Por qué tener en cuenta la dimensión afectiva?}

¿Por qué prestar atención a la dimensión afectiva en el contexto de la enseñanza? Pues porque tiene un gran poder e influencia en el proceso de aprendizaje. Tener en cuenta la dimensión afectiva, hace que la enseñanza sea mucho más humana y eficaz.

Desde los estudios en adquisición de la lengua de los años 1980, sabemos que las emociones negativas como el miedo o la ansiedad pueden dificultar en gran medida el aprendizaje, ya que los individuos se bloquean y no ponen en juego lo mejor de sí. Se aprende mejor en un ambiente libre de ansiedad y miedo. De manera paralela, las emociones positivas pueden facilitar, potenciar y hacer más eficaz el proceso de aprendizaje, por lo que resulta lógico conocerlas y estimularlas. Dentro de la dimensión afectiva, se pueden resaltar tres factores emocionales positivos fundamentales, que son la autoestima, la empatía y la motivación.

Sin embargo, debemos ser cuidadosos y no confundir el hecho de prestar atención a la dimensión afectiva en el aula con simplemente ser amables o transmitir a los estudiantes que todo lo que hacen está bien, incluso si no es el caso. Potenciar la autoestima y la confianza de los estudiantes se relaciona con "crear un ambiente de apoyo donde animamos a los alumnos a trabajar para alcanzar su potencial" (Arnold, 2015, p. 151). Hay que ser rigurosos para lograr un aprendizaje eficaz, que depende, en gran medida, de tener muy claro el objetivo de aprendizaje.

\section{Cognición y emoción}

Gracias a las investigaciones desarrolladas a finales del siglo XX y principios del XXI en neurociencia y psicología de la educación, sabemos que el hecho de dedicar atención al importante factor afectivo del aprendizaje no tiene por qué significar que dejemos de lado lo cognitivo. Ambos aspectos no están en oposición. Al contrario, si conseguimos aunar ambos factores, el proceso de aprendizaje puede construirse sobre unos cimientos más sólidos y resultará más eficaz.

La razón y la emoción están ligadas de forma inseparable en nuestra mente, son funciones complementarias. Si nuestra parte cognitiva es la que procesa la información, fija nuestros objetivos y planifica la actuación a seguir, es el lado emocional el que nos ayuda a implicarnos, a encontrar la motivación y a trabajar para conseguir alcanzarlos (Arnold, Foncubierta, 2019, p. 25). De este modo, la colaboración de ambas partes logrará mejores resultados. Asimismo, se deben tener en cuenta los factores físicos, como las condiciones ambientales de los estudian- 
tes, su bienestar y salud, su comodidad en el aula o la atención a la inteligencia cinestésico-corporal.

Se trata de considerar al aprendiente de forma holística, aunando lo cognitivo, lo emocional y lo físico. Así, si estimulamos los factores afectivos positivos podemos facilitar en gran medida el proceso de aprendizaje de las lenguas, ayudando a que el cerebro funcione de manera óptima e integrada.

Cabe recordar, como lo hace Arnold (2015, p. 151), que el éxito en el aprendizaje de lenguas depende menos de materiales, técnicas y análisis lingüísticos y más de lo que sucede dentro de y entre las personas en el aula. Al hablar de lo que sucede dentro de las personas en el aula, se refiere a los factores individuales como la autoestima, el auto-concepto, la ansiedad, las actitudes, los estilos de aprendizaje o la motivación. Por otra parte, cuando hablamos de lo que sucede entre las personas en el aula, nos referimos a los factores relacionales que se dan entre el profesor y los alumnos, entre los propios alumnos e incluso entre alumnos y la lengua y la cultura meta. Todos estos factores son fundamentales para el aprendizaje.

La neurociencia ha descubierto que la emoción es esencial para potenciar la curiosidad, la atención, la creatividad, la implicación personal, la memoria a largo plazo y, elemento fundamental, la motivación.

\section{Factores socioemocionales en el aprendizaje: la motivación}

Hace tiempo que sabemos, y constatamos en nuestra experiencia diaria, que sin motivación no hay aprendizaje. La motivación es, pues, un factor socioemocional fundamental, el motor que nos hace querer aprender. Ruiz Martín (2019, p. 160) la define como "un estado emocional que nos impulsa a emprender y mantener una conducta con un objetivo determinado".

Ya en el Marco Común Europeo de Referencia (MCER 5.3.1) se menciona la competencia existencial (saber ser), que incluye actitudes, motivaciones, valores, creencias y factores personales. Y más adelante, el MCER se refiere a la motivación como uno de los factores afectivos que influyen en el momento de realizar una tarea, junto con la empatía, la implicación, la actitud y el estado. Se hace una distinción entre motivación intrínseca y extrínseca. Como dice el propio MCER:

"Es más probable que una tarea se realice con éxito cuando el alumno se encuentra totalmente implicado; un nivel alto de motivación intrínseca para llevar a cabo la tarea provocada por un interés concreto por la tarea en sí o al percibir la importancia que pueda tener, por ejemplo, para las necesidades de la vida real o para la realización de otra tarea relacionada (la interdependencia de unas tareas con otras) fomenta una mayor implicación del alumno; la motivación extrínseca puede también desempeñar un papel importante cuando, por ejemplo, hay presiones externas para completar la tarea con éxito (por ejemplo: para recibir elogios, para no quedar mal, o, simplemente, por razones de competitividad)". (MCER, 7.3.1.2) 
Ambos tipos de motivación serán esenciales para fomentar un aprendizaje más activo.

\section{La motivación en los aprendientes de español como lengua extranjera}

Si bien es cierto que, en nuestro mundo actual, el inglés es la lengua extranjera más utilizada como lingua franca en muchos contextos, cabe no obstante preguntarse cuál es la motivación de los estudiantes para acercarse a otras lenguas, y, en concreto en el caso que nos ocupa, al español.

En primer lugar, podemos decir que el número de estudiantes de español es creciente en los últimos años. Por ejemplo, en Bélgica, país con una situación lingüística compleja y con tres lenguas oficiales (neerlandés, francés y alemán), el español constituye sin embargo la cuarta lengua extranjera más estudiada por los alumnos belgas (Pomar González, 2007). Asimismo, es la cuarta lengua más estudiada a nivel europeo.

La motivación extrínseca puede derivar de la importancia creciente del español en el mundo que, con casi 443 millones de hablantes nativos en el mundo, es la segunda lengua más hablada en el mundo después del chino mandarín y por delante del inglés. Asimismo, es la tercera lengua más utilizada en internet, por detrás del inglés y del chino. Lengua oficial en 21 países, presente en varios continentes, el español es percibido por nuestros estudiantes como una lengua útil e importante en el mundo. Según las cifras proporcionadas por el Instituto Cervantes (2019), cerca de 22 millones de personas estudian español como lengua extranjera.

En cuanto a la motivación intrínseca, puede explicarse por las connotaciones positivas que despierta esta lengua, el interés por América latina o por España, el gusto por los viajes o las culturas diversas o, en ocasiones, la percepción inicial y la creencia de que no es una lengua demasiado complicada de aprender, especialmente para estudiantes cuya lengua materna pertenece a la familia de las lenguas romances. Esta última creencia es en realidad un arma de doble filo ya que, si bien puede atraer en un principio a un gran número de estudiantes tentados por esta supuesta facilidad, el contraste con la dificultad real de la lengua puede desmotivar a los alumnos perezosos.

Por último, el hecho de que el español sea, en la mayoría de los casos, una lengua optativa y no obligatoria en el desarrollo de los currículos escolares hace que el nivel inicial de motivación de los estudiantes que encontramos en las aulas sea en general bastante alto. 


\section{Estrategias de motivación basadas en principios afectivos}

Como venimos diciendo, uno de los factores socioemocionales más importantes en el contexto de la enseñanza es la motivación.

Independiente de la motivación intrínseca o extrínseca que el alumno traiga consigo, y sabiendo, como explica Dörnyei (2001b, p. 51), que "la motivación para aprender, igual que la capacidad para adquirir el lenguaje, es un rasgo innato de la especie humana", la docencia tendría que colaborar de manera sustancial en el mantenimiento e incremento de la motivación, que tan fundamental resulta para el aprendizaje. De ahí que lleguemos a la pregunta fundamental: ¿cómo se puede fomentar esta motivación y hacerlo de manera sostenible? Los principios afectivos pueden ser de gran ayuda para orientarnos en esta tarea. Arnold y Foncubierta (2019, p. 31) adelantan ya que ["e]s posible que lo necesario para desarrollar y sostener la motivación en el aula radique más en que el alumno tenga control sobre su aprendizaje, posea un sentido de valores y propósito, conserve su autoestima y experimente el sentimiento de éxito".

En este sentido, y a partir de los estudios y propuestas de Dörnyei (2001a) podemos adelantar siete estrategias motivacionales generales basadas en principios que toman en cuenta los factores afectivos del aprendizaje.

1. La primera estrategia es la creación de una atmósfera relajada en el aula, con bajos niveles de ansiedad.

2. En segundo lugar, construir oportunidades para que los aprendientes experimenten el sentimiento del éxito, y aumentar de este modo su confianza. Hay una relación directa entre la confianza y la competencia, con lo que favorecer la primera ayuda a mejorar la segunda.

3. Considerar al alumno holísticamente: con sus aspectos cognitivos, emocionales y físicos.

4. Proporcionar experiencias personalmente significativas, $y$, si es posible, experiencias memorables de aprendizaje.

5. Favorecer y desarrollar la autonomía del aprendiente.

6. Utilizar los conocimientos previos y los recursos propios del estudiante.

7. Incorporar la elección en las clases, ya que la elección es intrínsecamente motivadora. Todos preferimos hacer algo que hemos elegido en lugar de una tarea obligatoria o impuesta. Además, la elección ayuda a desarrollar el sentimiento de autonomía y a responder a la diversidad de los estudiantes.

Otra importante estrategia motivacional, si podemos llamarla así, es el uso del aprendizaje cooperativo, que favorece muchas de las siete mencionadas anteriormente. Este tipo de aprendizaje tiene en cuenta lo relacional, y consigue que los alumnos disfruten creando algo juntos. Efectivamente, en primer lugar, trabajar 
en pequeños grupos cooperativos desarrolla la responsabilidad social en una comunidad de aprendientes y la inteligencia interpersonal, que se caracteriza por la capacidad de comprender y responder efectivamente a los demás. En segundo lugar, los trabajos de grupo requieren diferentes habilidades, con lo que, al trabajar de manera cooperativa, se reconoce la diversidad de los estudiantes. Además, de este modo se favorece el aprender haciendo (learn by doing) y el aprendizaje activo tal como lo define Ruiz Martín (2019), es decir, aprender pensando (learn by thinking), y ayuda a evocar lo aprendido al colaborar con otros compañeros que tengan habilidades y conocimientos diversos. Por último, el aprendizaje cooperativo estimula factores afectivos positivos como la implicación y la autoestima.

En lo que respecta a la motivación de los docentes, Dörnyei (1998) recoge muchos de estos principios motivacionales, que son bidireccionales, dado que tener un profesor motivado favorece notablemente la motivación en los aprendientes y viceversa. Además, muchas de las condiciones se refieren al aula, al ambiente y al modo de abordar el proceso de enseñanza-aprendizaje, que es el terreno común. Así, es interesante recordar los "diez mandamientos para motivar a los aprendientes de lenguas" de Dörnyei y Csizér (1998), que recogen muchos de estos principios y coinciden en gran medida con las estrategias mencionadas anteriormente:

1. Da ejemplo personal con tu actitud y con tu propio comportamiento.

2. Crea un ambiente relajado y agradable en el aula.

3. Presenta las tareas y actividades adecuadamente. Dar instrucciones claras y precisas es fundamental para evitar malentendidos y frustraciones inútiles.

4. Desarrolla una buena relación con los estudiantes.

5. Incrementa la auto-confianza lingüística de los estudiantes.

6. Haz las clases de lengua interesantes.

7. Favorece la autonomía del estudiante.

8. Personaliza el proceso de aprendizaje.

9. Incrementa la orientación dirigida a objetivos de los estudiantes.

10. Familiariza a los aprendientes con la/s cultura/s de la lengua meta.

De manera general, una vez consideradas las diversas propuestas de los especialistas en el tema, para lograr despertar la motivación podemos resumir en una serie de recomendaciones que intentaremos después relacionar con una actividad que pueda concretarlas.

En primer lugar, nos queda claro que es importante crear un ambiente libre de ansiedad, donde todas las personas que están en el aula puedan sentirse a gusto y libres para expresarse, de modo que el cerebro funcione de manera óptima, sin miedo ni estrés, y los estudiantes puedan implicarse y ser creativos. 
Concebir actividades significativas, que resulten relevantes e interesantes para los aprendientes, que tengan que ver con su vida, con su mundo, pero que a la vez les abran la visión a un mundo desconocido, a la(s) cultura(s) de la lengua meta. Es necesario que esas actividades tengan un nivel adecuado, no demasiado fácil porque sería aburrido, pero tampoco demasiado difícil porque resultaría frustrante. El justo medio sería lograr proponer un reto accesible. Para ello, conviene partir del conocimiento previo y de los recursos propios de los alumnos, y fomentar su autonomía, es decir, favorecer que tomen un papel activo, se impliquen y se hagan responsables de su aprendizaje.

¿Cuál sería entonces el papel del profesor? El profesor influye - consciente o inconscientemente -en que los alumnos quieran saber (Alonso Tapia, 1991), ya que un profesor motivado favorece notablemente la motivación de los aprendientes, pero el alumno está en el centro del proceso de aprendizaje. Por ello, "el profesor no debe enseñar, sino ayudar a aprender. El que aprende es nuestro alumno y nuestra tarea es facilitar ese aprendizaje" (Morales Vallejo, 2006, p. 3).

\section{Tareas y actividades: un festival de cortometrajes}

Todos estos principios y estrategias de motivación pueden aplicarse en el aula de lenguas de infinitas maneras. Nosotros aquí quisiéramos avanzar una propuesta que, en nuestra opinión, engloba muchos de los aspectos mencionados anteriormente.

Se trata de organizar un festival de cortometrajes, escritos y realizados por los propios estudiantes en grupos de cuatro personas. La duración máxima de los cortometrajes es de diez minutos por grupo, y la actividad ocupa un cuatrimestre desde su inicio, acompañada por tareas de preparación y acompañamiento en torno al cine, realizadas en el aula.

Los pasos de la tarea son los siguientes: en primer lugar, los estudiantes deben escribir el guion en grupo, poniendo un título a su película y añadiendo una sinopsis (tipología textual que se trabaja en clase). Después, lo graban con ellos mismos como personajes, directores, etc. Por último, se fija una fecha para la proyección en clase de todos los cortos, y se otorgan los "Premios Goya" (premios cinematográficos otorgados por la Academia de las Artes y las Ciencias Cinematográficas de España), votados por los propios estudiantes, con las categorías de mejor actor y actriz, mejor película, mejor vestuario, mejor B.S.O, etc. Son los estudiantes los que negocian y deciden, de manera anticipada, los premios que se otorgarán, y son ellos mismos el jurado que vota tras el visionado. El docente acompaña, coordina, recuenta los votos y puede proclamar a los nominados y a los ganadores de cada categoría en una ceremonia de entrega de premios.

Esta actividad resulta muy motivadora por la implicación y creatividad que pone en movimiento. En primer lugar, es un trabajo colaborativo, por lo que se maxi- 
mizan las sinergias de grupo, se atiende a la diversidad y se pueden aprovechar y valorar las capacidades individuales de cada estudiante. Por ejemplo, en algunos grupos hay quien sabe componer música, o tiene facilidad para el grafismo, o talento literario, y les resulta muy satisfactorio poner estas habilidades al servicio de su grupo. Además, pueden ayudarse mutuamente si tienen niveles heterogéneos en las diversas competencias o actividades comunicativas de la lengua.

Este tipo de proyecto reduce mucho la ansiedad en el aula y fomenta un ambiente distendido de trabajo en grupo e individual. Los estudiantes sienten que el resultado final es "suyo", se apropian de cada etapa del proceso y se implican personalmente. En clase están atentos y participan en las tareas facilitadoras, porque saben que les serán útiles para su propio trabajo. Se trata de una actividad significativa, en la que ellos pueden reflejar su mundo, sus preocupaciones y su imaginación. Pueden elegir cualquier género cinematográfico, desde la comedia al drama pasando por el documental, la película de terror o el thriller. El amplio grado de elección aporta asimismo un mayor nivel de motivación y de implicación, ya que sienten que pueden inventar y expresar lo que desean. Esto queda confirmado por el feedback recibido cada año de los estudiantes, que es extremadamente positivo $\mathrm{y}$ alentador, $\mathrm{y}$ en el que resaltan que han disfrutado mucho aprendiendo gracias a esta tarea, que la consideran una "excelente idea" para aprender y para poner en práctica lo aprendido de una manera global, eficaz y divertida, que además potencia su creatividad.

Definitivamente, esta tarea fomenta la autonomía del estudiante y su orientación hacia objetivos. Aprenden haciendo, siendo y disfrutando, y definitivamente ayuda a incrementar la autoestima y la confianza en sí mismo, sobre todo en el momento catártico y muy gratificante de la ceremonia final de los "Premios Goya", que muestran el reconocimiento y apreciación de los propios compañeros.

\section{Conclusión}

El cambio de paradigma que proponemos para lograr un proceso de adquisición de la lengua realmente eficaz supone proporcionar experiencias de aprendizaje memorables, basadas en principios humanísticos, que consideren al aprendiente de manera holística y que sepan integrar los aspectos cognitivos, afectivos y físicos. Para realizar estas experiencias, y que sean realmente memorables y efectivas, tenemos que tener en cuenta las estrategias de motivación que incorporan la atención a la dimensión afectiva del aprendizaje. Entre ellas, la aplicación de un aprendizaje cooperativo es un instrumento útil en la práctica, ya que se trabaja en pequeños grupos para realizar tareas significativas. En este tipo de proyectos, como, por ejemplo, la organización en el aula de un festival de cortometrajes, se fomentan los factores afectivos positivos como la implicación del estudiante, su autoestima y, de manera fundamental, su motivación. De este modo, podemos lograr un aprendizaje adaptado a la diversidad e individualidad, motivador, eficaz, 
completo y orientado a resultados, integrando a la vez las dimensiones de ser, hacer y disfrutar.

\section{Bibliografía}

Alonso TAPIA, J. (1991). Motivación y aprendizaje en el aula: Cómo enseñar a pensar. Madrid: Santillana.

ARnoLD, J. (1999). Affect in language learning. Cambridge: Cambridge University Press.

ARNoLD, J. (2015). Explorando la dimensión afectiva de la enseñanza de ELE, en VV.AA. La formación del profesorado de español. Innovación y reto, Barcelona: Editorial Difusión, Cuadernos de didáctica.

ARNold, J., \& FonCuBiERTA, J. M. (2019). La atención a los factores afectivos en la enseñanza de ELE: Claves para la enseñanza de español. Madrid: Edinumen.

ConSEjo DE EuRoPA. (2002). Marco común europeo de referencia para las lenguas: aprendizaje, enseñanza, evaluación. Madrid: MECD y Anaya.

DöRNYEI, Z., \& CSIZER, K. (1998). Ten commandments for motivating language learners: results of an empirical study. Language Teaching Research, 2, 203-229. http://dx.doi.org/10.1177/ 136216889800200303 Consultado el 12 回回.

DöRNYEI, Z. (2001a). Motivational strategies in the language classroom. Cambridge: Cambridge University Press.

DöRNYEI, Z. (2001b). New themes and approaches in L2 motivation research. Annual Review of Applied Linguistics, 21, 43-59.

MoRALES VALLEJo, P. (2006). Implicaciones para el profesor de una enseñanza centrada en el alumno. Miscelánea Comillas: Revista de Ciencias Humanas y Sociales, 64(124), 11-38.

Pomar GonzÁLEZ, R. (2007). El español en Bélgica. Enciclopedia del español en el mundo. Barcelona: Plaza y Janés.

Ruiz MARTín, H. (2019). ¿Cómo aprendemos? Una aproximación científica al aprendizaje y la enseñanza. Barcelona: Grao.

SPOLSKY, B., \& HulT, F. M. (Eds). (2008). The handbook of educational linguistics. Oxford: Blackwell Publishing.

TRujILlo SÁEz, F., (2015). De la provisión de servicios a la creación de experiencias: la necesidad de un nuevo marco para la enseñanza y el aprendizaje (de lenguas) en el siglo XXI en VV.AA. La formación del profesorado de español. Innovación y reto. Barcelona: Editorial Difusión, Cuadernos de didáctica.

YUN DAI, D., \& STERNBERG, D. J. (Eds.) (2004). Motivation, emotion and cognition. Integrative perspectives on intellectual functioning and development. Mahwah: Lawrence Erlbaum Associates, Publishers.

\section{Autor}

Beatriz Calvo, Université libre de Bruxelles, e-mail: bcalvoma@ulb.ac.be

The author is Professor of Spanish Language and Didactics at the Université libre de Bruxelles and has a $\mathrm{PhD}$ in Languages and Literature. Her broad teaching and teacher training experience includes collaborations with several universities, Instituto Cervantes and other institutions. Her research deals with applied linguistics, L2 motivation, creativity in language learning, affect in language teaching, Spanish literature, Francophone literature and Comparative literature. She is also the author of learning materials (Spanish for Academic Purposes), a novel (La Jaula Invisible) and three short stories (Nocturno, Un sueño de sueños and Últimas voluntades), published in Spain. 